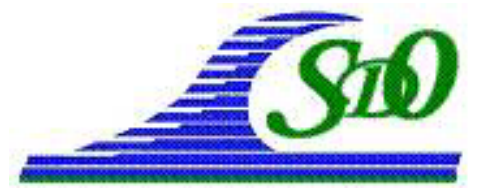

XI ìmes Journées Nationales Génie Côtier - Génie Civil

Les Sables d'Olonne, 22-25 juin 2010

DOI:10.5150/jngcgc.2010.067-A C Editions Paralia CFL

disponible en ligne - http://www.paralia.fr - available online

\title{
Le suivi environnemental du dragage de Vavouto (Nouvelle-Calédonie). Un chantier de dimension mondiale à la porte d'un site classé au patrimoine mondial
}

\author{
Michel ALLENBACH ${ }^{1}$, Morgan MANGEAS ${ }^{2}$, TOURAIVANE ${ }^{1}$
}

1. Université de la Nouvelle-Calédonie, PPME, EA 3325, BPR4, 98851 Nouméa Cedex, Nouvelle Calédonie.

allenbach@univ-nc.nc;touraivane@univ-nc.nc

2. IRD Centre de Nouméa, US Espace, BP A5, 98848 Nouméa Cedex,

Nouvelle Calédonie.

morgan.mangeas@ird.noumea.nc

\section{Résumé :}

La construction d'une usine métallurgique de dimension mondiale, consacrée à la transformation du minerai de nickel, nécessite l'ouverture d'un important chenal de navigation (4500 m de longueur et $7300000 \mathrm{~m}^{3}$ de déblais) dans le lagon nord-ouest néo-calédonien. Les travaux de dragage sont réalisés sur un domaine lagonaire situé à proximité immédiate de zones coralliennes récemment classées au patrimoine mondial de l'humanité en raison de la biodiversité exceptionnelle de l'écosystème récifal calédonien. La communication présente rapidement le plan de suivi environnemental qui intègre un réseau de suivi en temps réel de la turbidité ambiante et des paramètres physico-chimiques de la qualité de l'eau et des sédiments, ainsi qu'un important volet biologique. Elle détaille les mesures relatives au suivi de la turbidité ambiante. Sont ensuite exposés les résultats des contrôles environnementaux réalisés et l'intérêt d'une démarche originale pour assurer leur restitution aux populations autochtones riveraines du site: la création du CEK (comité environnemental du Koniambo). L'enjeu est essentiel pour le pays : contribuer au rééquilibrage économique inscrit dans la loi organique et les accords de Nouméa, dans le respect d'un environnement fragile et des pratiques sociétales des populations vivant dans la zone impactée. La dernière partie traite de l'intérêt de l'utilisation des bases des données interopérables pour la gestion environnementale et la restitution des résultats des suivis environnementaux dans des contextes sensibles. Elles facilitent la communication entre techniciens et vers l'extérieur. Elles rendent plus aisées les méthodes de contrôle à mettre en œuvre pour analyser les données transmises par les opérateurs en charge du suivi.

Mots-clés :

Dragages - Turbidité - Plan de suivi environnemental - Nouvelle-Calédonie Ecosystème récifal - Base de données interopérables - Développement durable 


\title{
Thème 4 - Vulnérabilité du littoral
}

\begin{abstract}
:
In the northern part of New-Caledonia, the installation of a factory dedicated to the nickel ore processing requires to dredge a navigation channel of $4500 \mathrm{~m}$ long with $7300000 \mathrm{~m}^{3}$ of extracted materials. The dredging process has taken place very near of sites inscribed recently in the world heritage list of UNESCO because of the exceptional biodiversity of their reefs and associated ecosystems. First, this paper introduces the framework of the environmental supervision which includes a real-time monitoring of the physico-chemical parameters of the water and the sediments, as well as an important assessment system of biological observations. The measurements relative to the monitoring of turbidity are detailed and analysed. Then, the results of the environmental monitoring and the interest of the chosen approach are explained. Especially, an environmental committee of Koniambo (named CEK) has been set up in order to assure that all the monitoring observations are communicated to the local populations. This action is a crucial stake for the country. The factory can contribute to the economic rebalancing between northern and southern parts of the island as registered in previous nationwide agreements, but this should be done in the respect of the fragile environment and the societal practices of the populations which live in the impacted zone. The last section deals with the interest of the use of interoperable data bases for the environmental management. Some hints are proposed about analysis methods, modelling and information flow of the environmental monitoring within this sensitive context. They are meant to facilitate the communication between operators, technicians, engineers and consultants. The proposed methods make also easier the check of the procedures and the analysis of the data by the experts in charge of the monitoring.
\end{abstract}

\section{Key-words:}

Dredging process - Turbidity - Environmental supervision - New Caledonia - Reef ecosystem - Interoperable database - Sustainable development

\section{Introduction. Présentation générale du "Projet Koniambo"}

Sur la côte nord-ouest de la Grande Terre néo-calédonienne, dans le Pacifique sudouest, la construction d'une usine métallurgique de transformation du minerai de nickel est en phase opérationnelle et devrait se terminer en 2013. Le chantier, de dimension mondiale, impacte la zone "VKP" du nom des trois communes (Voh, Koné, Poimbout) sur lesquelles sont implantées l'essentiel des infrastructures directes et indirectes d'un programme industriel clef des "Accords de Nouméa" : le projet Koniambo, du nom du massif minier qui va être mis en exploitation pour alimenter l'usine. Les infrastructures à créer (usine, base-vie, centrale thermique, site minier) génèrent des besoins significatifs en matières premières (charbon, hydrocarbures, modules préfabriqués de l'usine) à importer et la nécessité, en retour, d'exporter le produit fini (ferro-nickel). Dans le contexte insulaire océanien, la voie maritime s'imposait pour ces transports et la décision a été prise de construire un port. Le site portuaire choisi (Vavouto) nécessite 
l'ouverture d'un chenal de navigation pour permettre l'accès sécuritaire au site à des navires pouvant atteindre 50000 TPL.

\section{Descriptif rapide des travaux de dragage}

\subsection{Données générales sur les travaux}

Confiés à la société "Jan de Nul", l'une des "majors" du dragage mondial, les travaux ont débuté en août 2008 et doivent se terminer en avril 2010. Ils visent la réalisation d'un chenal de navigation (12 mètres de profondeur, $120 \mathrm{~m}$ de large, $4,5 \mathrm{~km}$ de long) entre le port industriel associé à l'usine et la passe du Duroc qui permet la sortie en haute mer, au-delà du récif-barrière. Les études préliminaires ont estimé à $7300000 \mathrm{~m}^{3}$ le volume des déblais à extraire dans des matériaux, variables selon les zones, depuis des silts, des boues et des sables jusqu'à des constructions coralligènes indurées. Ces caractéristiques ont nécessité la mise en œuvre sur le terrain de deux types de dragues sur les 13 zones caractérisées par les travaux de reconnaissance (figure 1). Il a été utilisé, d'une part, une DAET (drague autoporteuse à élinde traînante) d'une capacité de $3700 \mathrm{~m}^{3}$, et d'autre part, une DE (drague excavatrice dotée d'un godet de pelle de $40 \mathrm{~m}^{3}$, assistée de deux barges autopropulsées de $3700 \mathrm{~m}^{3}$ chacune. La DAET a été affectée au dragage des sédiments fins meubles et la $\mathrm{DE}$ à celui des sédiments consolidés. Le matériel dragué sur la zone littorale pour atteindre la bathymétrie de projet est transporté vers la zone d'immersion des déblais, située à l'extérieur du récifbarrière, sur des fonds supérieurs à $1000 \mathrm{~m}$. Le site d'immersion des déblais (suspension concentrée pour la DAET et sédiment semi-solide saturé en eau pour les barges associées à la $\mathrm{DE}$ ) est un carré de $1 \mathrm{~km}$ d'arête, situé à une distance de $5 \mathrm{~km}$ de la pente externe du récif-barrière, au sud-ouest de la passe de Duroc. Sa localisation, réputée sans incidence sur la biodiversité récifale, a fait l'objet de nombreuses modélisations validées par INERIS, tiers-expert de l'étude d'impact environnementale générale du Projet Koniambo. Les conclusions du tiers expert, rendues en 2005, ont permis la prise de l'arrêté $n^{\circ}$ 180/2005 du 31/12/2005 portant autorisation domaniale de réaliser des travaux de dragage, d'endigage et d'immersion des déblais sur le domaine public maritime de la province Nord.

Un PGE (plan de gestion environnementale) de l'impact des travaux a été demandé par INERIS dans ses recommandations. Compte tenu du spectre large des aspects traités par le PGE, la communication cite de façon très succincte les caractéristiques générales de ce suivi environnemental et focalise sur un seul enjeu : la turbidité ambiante. 


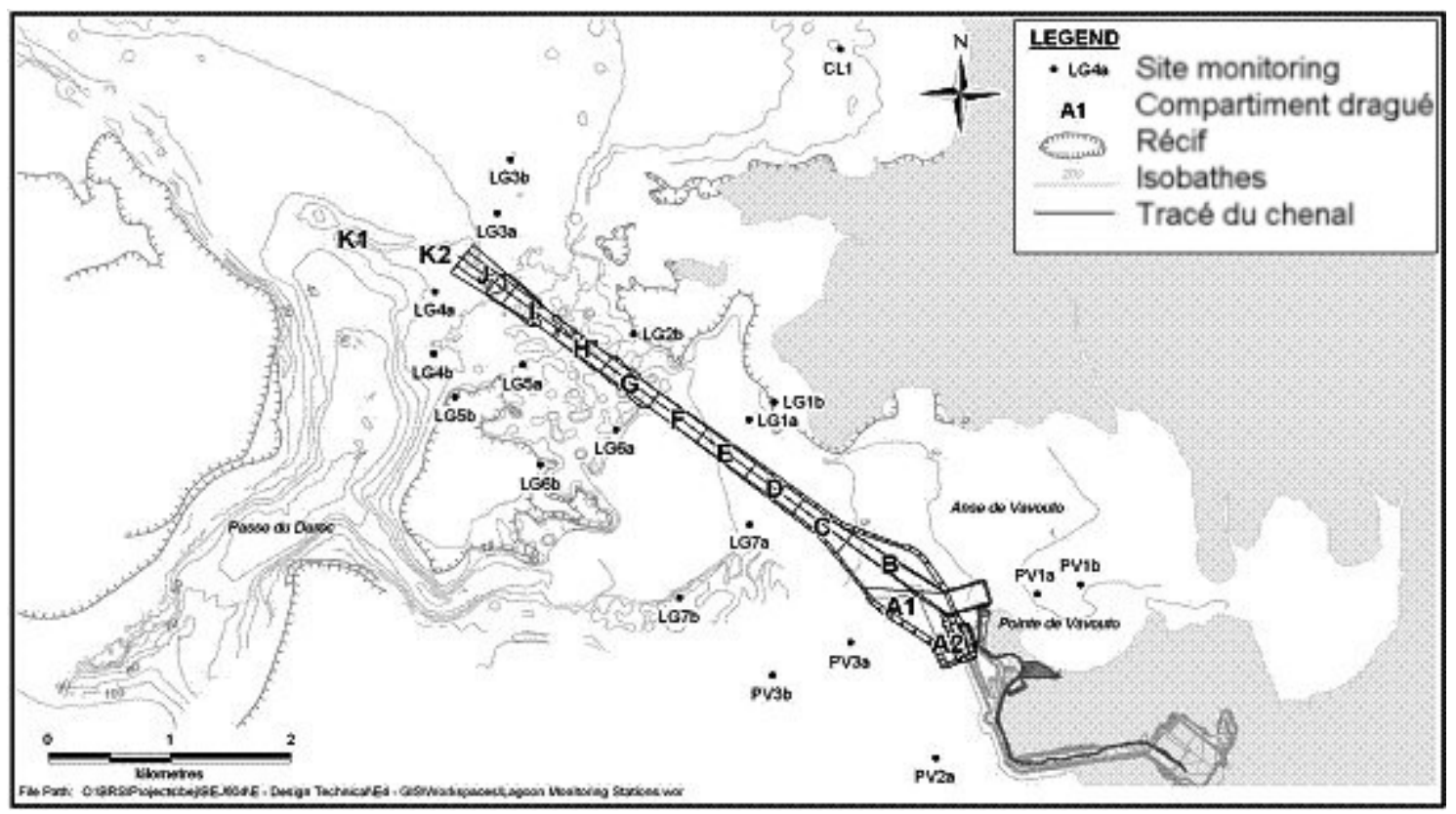

Figure 1. Le tracé du chenal et les 13 zones de travail (d'après KBR-Rapport BEJ604).

\subsection{Un contexte environnemental sensible}

Les travaux intéressent un domaine lagonaire situé à proximité immédiate de zones récemment classées (2008) au patrimoine mondial de l'humanité. Réalisée à $85 \%$ aujourd'hui, l'avancée des travaux permet de dégager une analyse critique sur le degré d'efficacité du programme de suivi environnemental mis en place en regard des attentes exprimées. Le projet industriel de KNS (Koniambo Nickel Society) est, en effet, au centre d'un pari essentiel pour le pays : celui du développement durable à réussir dans le respect d'un environnement fragile et des pratiques sociétales des populations autochtones qui vivent dans la zone impactée. Le fort attachement culturel des mélanésiens envers leur milieu naturel les rend très attentifs au respect de l'environnement. Les contraintes sur l'industriel en matière de respect de l'environnement sont donc fortes du côté des populations locales. Elles sont également fortes du côté des associations et organisations internationales qui œuvrent sur l'environnement. Le classement du récif calédonien impose des contrôles stricts et des pratiques exemplaires en vue de la sauvegarde des sites naturels. Le peu d'échos qui se sont fait jour jusqu'ici, au sujet du chantier du dragage, démontre le succès de l'approche suivie: politique de communication claire, partenariat attractif avec les populations locales, création du comité environnemental du Koniambo. 


\section{Le plan de suivi environnemental du dragage de Vavouto (volet turbidité)}

\subsection{Généralités sur la démarche suivie}

A la fin 2007, le plan de gestion environnementale pour le dragage du chenal demandé par l'INERIS en 2005, a été produit par KBR (Kellog Brown Root Ltd, 2007) pour le compte de KNS et présenté à la province nord. Celle-ci a mandaté un tiers-expert, l'université de la Nouvelle Calédonie (UNC), pour le valider et après remise des conclusions de ce dernier (ALLENBACH, 2008), les travaux ont débuté en août 2008. Le plan distingue 6 enjeux d'importance variable: la qualité de l'eau, de l'air, l'écologie (flore et faune), le bruit, la gestion des déchets et des substances dangereuses.

\subsection{Les mesures relatives au contrôle de la turbidité ambiante à l'intérieur du lagon}

Seul le volet contrôle de la turbidité ambiante à l'intérieur du lagon sera détaillé dans le cadre de la communication. Il s'agit, d'une part, de l'effet induit le plus apparent, susceptible non sans raison, de fédérer les craintes et les oppositions aux travaux et d'autre part, du paramètre le plus pénalisant pour l'écosystème corallien. Le tableau 1 permet de comparer la proposition initiale produite par KBR pour le compte de KNS et les mesures retenues par l'autorité provinciale après la tierce-expertise UNC. Le système de bouées fixes mis en place pour mesurer la turbidité en temps réel (radiotransmission des données recueillies, au pas d'une mesure/3mn) et de façon plus classique (stations fixes autonomes travaillant au pas d'une mesure $/ 30 \mathrm{mn}$, déchargées hebdomadairement) est constitué par 2 bouées OMC-045-B-1200 (mesure de la turbidité et de la houle) et 8 bouées OMC-45-B-250 (mesure de la turbidité). Les bouées ont été équipées de sondes multi-paramètres de type YSI 6920 et HYDROLAB MS ET DS 5. Le travail de terrain est assuré par deux prestataires sous la supervision de HT (Hatch-Technip) et KBR.

\subsection{Les résultats du suivi}

Le traitement des 10 premiers mois de données montre qu'il n'y a pas eu de dépassement significatif des seuils autorisés ( $>$ à 70 NTU sur 1 heure ou $>$ à 30 NTU sur 3 jours). Les seuls dépassements notés sont liés à des problèmes de salissure (biofooling) des capteurs, solutionnés par des interventions de nettoyage des plongeurs et des équipes de maintenance. Des dysfonctionnements (signal haché) ont également été notés pour les données transmises par radio, résolus par le déplacement des relais. 
Tableau 1. Comparatif des mesures préconisées et retenues.

\begin{tabular}{ll}
\hline Mesures proposées par KNS & Mesures mises en place \\
\hline Surveillance visuelle journalière des & Mise en place d'un système de bouées \\
panaches sédimentaires ; & d'acquisition et de transmission en temps \\
Mesures toutes les 30 minutes de & réel (pas de 3 minutes) des valeurs de \\
la turbidité de l'eau et & turbidité de l'eau ; \\
déchargement & Mesures en parallèle, toutes les 30 minutes \\
hebdomadaire des données & de la turbidité de l'eau et déchargement \\
enregistrées par bouées & hebdomadaire des données. \\
spécifiques. & Mesures directes manuelles à proximité de \\
& la zone de dragage, \\
& Mise en place d'un système d'alerte se \\
& déclenchant en cas de dépassement des \\
& valeurs critiques retenues par le projet. \\
\hline
\end{tabular}

\subsection{La communication environnementale}

L'autorité provinciale et l'industriel se sont accordés pour instituer une cellule d'interface entre la population et le projet Koniambo : le CEK. Composée d'élus, de scientifiques, de représentants du monde associatif et coutumier, directement intégrée dans le projet qui la subventionne, elle est animée par des techniciens environnementalistes, salariés par elle. Elle intervient efficacement pour le respect des engagements pris ou pour l'obtention de compensations environnementales en cas de dysfonctionnements. Elle anime les séances de restitution des résultats du suivi, crée les espaces de discussion souhaitables. La démarche est incontestablement un succès puisqu'au bout de 18 mois et à moins de 3 mois de son terme, le programme ne souffre d'aucune critique significative.

\section{Les améliorations potentielles de la démarche}

L'analyse et la transmission des résultats auraient pu être facilitées si le recueil, l'organisation, le stockage et la diffusion de la donnée, notamment celle provenant des capteurs, avaient été mieux organisés. Une réflexion associant l'ensemble des acteurs (bureaux d'étude, compagnie minière, experts et collectivités), menée en amont des travaux, aurait donné une plus grande cohérence dans le processus allant de l'acquisition à l'analyse. Il ne s'agit pas ici de tenter d'uniformiser les productions de chaque acteur, sachant que chacun possède ses outils et sa manière de fonctionner, mais plutôt de fournir des recommandations afin que ces productions puissent s'insérer dans un système d'information directement exploitable à des fins d'analyse et de visualisation d'indices, voire d'outils d'aide à la décision. 


\section{XI $I^{\text {èes }}$ Journées Nationales Génie Côtier - Génie Civil}

Les Sables d'Olonne, 22-25 juin 2010

\subsection{L'intérêt de la création d'une base de données inter-opérables}

Diverses initiatives, tant européennes (INSPIRE) qu'internationales, (GMES, GEOSS) visent à fédérer les activités sur les systèmes d'information afin d'optimiser l'utilisation des infrastructures existantes et futures. L'objectif est de développer les moyens de collecte et de diffusion de données et de permettre leur intégration dans des projets de suivis environnementaux. L'évolution des Systèmes d'Informations Environnementaux (SIE) s'appuie sur des architectures distribuées et inter-opérables. L'intérêt de telles approches réside dans la facilité de partage et de diffusion des informations à différentes communautés d'acteurs (décideurs, services techniques, secteur privé et grand public). En particulier, les avancées technologiques permettent, de plus en plus facilement, de collecter des données au moyen de capteurs in-situ éloignés et de les contrôler à distance. Parallèlement, des initiatives de standardisation ont vu le jour pour favoriser : (a) l'utilisation d'applications ouvertes pour le traitement de l'information provenant des capteurs, (b) la coopération entre les fournisseurs de systèmes de capteurs, leurs gestionnaires et les "consommateurs" des données. Actuellement, la création de réseaux de capteurs distribués et facilement accessibles exige beaucoup de temps et d'efforts. Plusieurs organisations fournissent des systèmes d'acquisition de données, mais peu d'entre elles fournissent des interfaces et aucune n'offre des outils génériques normalisés. Aussi, l'OGC s'est donné pour objectif de publier des spécifications pour la structuration de la diffusion des données issues de capteurs de tous types hébergés et contrôlables par Internet. L'objectif des normes est de permettre la mise en place de réseaux de capteurs interrogeables à distance selon des protocoles publics. Les standards de la suite "Sensor Web Enablement" (SWE) proposés par l'OGC constituent un cadre riche pour normaliser l'acquisition, le stockage et la diffusion des informations; informations concernant aussi bien la description des capteurs euxmêmes que les données issues de ces capteurs. À titre d'exemple, les spécifications suivantes font partie de cette suite :

- Le langage "Sensor Markup Language" permet de définir dans un format commun la description des capteurs et des systèmes de capteurs. Les fonctions prises en charge relatives aux capteurs comprennent leur découverte, leur géolocalisation, le traitement des observations, leur programmation, les abonnements à des alertes.

- La spécification "Observations and Measurements" (O\&M) définit un modèle conceptuel de données pour organiser les bases de données relatives aux observations et mesures réalisées. Une observation est un événement situé temporellement et qui génère une certaine valeur correspondant à un phénomène observé. En dehors de ces deux valeurs, O\&M permet également de décrire d'autres propriétés comme le processus qui a permis d'obtenir ces valeurs, la position spatiale (primordiale pour des capteurs mobiles), la qualité de la mesure.

Notons toutefois que même pour les capteurs qui ne peuvent transmettre les mesures et être pilotés à distance selon les standards préconisés, il est important d'alimenter une 


\section{Thème 4 - Vulnérabilité du littoral}

base de données constituée des données recueillies par des méthodes traditionnelles. Cette base de données "tampon" permet de simuler une partie des comportements d'un capteur standardisé. La conformité à ces standards facilite également le croisement des données issues des capteurs réels ou simulés avec d'autres standards de l'Open GIS Consortium (OGC) largement répandus et dédiés à l'information géographique tels que "Web Map Service" (WMS) et "Web Coverage Service" (WCS).

En s'affranchissant des difficultés liées à la disparité des capteurs et en se basant sur des modèles de données reconnus, l'élaboration et l'utilisation d'un système d'information sont grandement simplifiées. Il est à souligner que cette étape est cruciale et souvent négligée ou sous-estimée habituellement; ce qui conduit à un surcoût de travail par la suite, à une quantité ou qualité de données inadéquates aux besoins, et in fine, à des difficultés quelquefois insurmontables pour analyser les données. Ce système d'information servira de socle pour développer, de manière générique, les outils de diffusion/visualisation mais également pour fournir des tableaux de bord thématiques synthétiques à des fins d'alerte, de concertation et de décision.

\subsection{L'intérêt d'un traitement optimisé de données hétérogènes naturalistes}

Dans cette étude, les données de turbidité étaient fournies sous forme de tableaux permettant d'opérer des statistiques de base afin d'en estimer la qualité, la fiabilité et la précision (détermination des moments d'ordre 1 et supérieur, fréquence, tendance et saisonnalité, estimation du bruit et détection des données aberrantes et/ou manquantes). Un travail de nettoyage et de vérification de la donnée a cependant été nécessaire. L'automatisation du processus était rendue difficile par l'éparpillement des données dans plusieurs fichiers ou supports ne respectant pas toujours le même format. Si les informations récoltées avaient été organisées dans une base de données structurée en suivant un protocole déterminé, avec des métadonnées associées suffisamment détaillées pour déterminer la provenance et les conditions de recueils, cette étape d'analyse exploratoire s'en serait trouvé grandement simplifiée. Ceci est d'autant plus essentiel dans le cadre d'études environnementales qui intègrent des données de nature hétérogène (quantitative continue ou discrète, qualitative ordonnée ou non...) et de qualité variable. Outre la qualification des données, un des objectifs fixé était de déterminer les valeurs journalières de turbidité dépassant un certain seuil déterminé par les experts et de croiser ces évènements avec d'autres tels que le nettoyage des capteurs (voir partie 3.3).

La possibilité d'exploiter une base de données structurée permettant d'effectuer des requêtes temporelles et spatiales croisées apparaît ici particulièrement intéressante et entraîne un gain de temps substantiel. Il est à noter aussi que la plupart des logiciels de statistiques évolués tels que $R$ (DRAY \& DUFOUR, 2007) autorise les requêtes sur les bases de données les plus courantes. Le triplet base de données/outils d'analyse/système d'information semble donc utile dans le cadre de ce plan de suivi environnemental, 


\section{XI $I^{\text {èes }}$ Journées Nationales Génie Côtier - Génie Civil}

Les Sables d'Olonne, 22-25 juin 2010

d'autant plus qu'il est prévu d'opérer d'autres analyses qui seront plus faciles à mettre en œuvre dans cette configuration. Il s'agit notamment d'évaluer les corrélations entre les différentes variables afin de calculer des interpolations et de modéliser leurs dynamiques spatio-temporelles. A partir de ces modélisations, des indicateurs pourront être construits. Ces indicateurs seront élaborés par les experts et les intervalles de confiance, les biais, et les précisions associées seront fournies. La dynamique des données sera aussi étudiée soit en mode univarié (dynamique d'une variable particulière telle que la turbidité dans notre cas) soit en mode multivarié (i.e. estimer la distribution spatiale et temporelle des sédiments en fonction de données de courantologie, météorologiques et autres). Cette étape nécessite l'utilisation de modèles mathématiques qui peuvent s'avérer complexes à mettre en œuvre (définition de la structure du modèle ainsi que le calibrage des paramètres) avec des risques d'obtenir des résultats avec une faible fiabilité. A partir des différents indicateurs et des sorties de modèle, des recommandations automatiques pourront être proposées afin d'aider les décideurs à mettre en place les mesures à prendre. Ces outils peuvent s'appuyer sur des méthodologies allant d'une combinaison linéaire simple des paramètres déterminants jusqu'à un processus complexe pouvant faire intervenir des concepts d'intelligence artificielle tels que les réseaux bayésiens (BORGELT \& KRUSE, 2002). Ces graphes probabilisés possèdent notamment des capacités permettant de prendre en considération simultanément les connaissances a priori des experts (à travers une structure de graphe) et l'information contenue dans les données. 
Thème 4 - Vulnérabilité du littoral

\section{Références bibliographiques}

ALLENBACH M. (2008). Projet Koniambo (travaux de dragage) - Analyse critique du plan de suivi environnemental. Rapport, université de la Nouvelle-Calédonie, 69 p.

BORGELT C., KRUSE R. (2002). Graphical Models: Methods for Data Analysis and Mining. Chichester, UK: Wiley. ISBN 0-470-84337-3.

DRAY S., DUFOUR A.B. (2007). The ade4 package: implementing the duality diagram for ecologists. Journal of Statistical Software 22(4), pp 1-20.

KBR -Kellog Brown Root Ltd- (2007). Plan de suivi environnemental des travaux de dragage, Rapport BEJ604-G-REP-001, 47 p. 